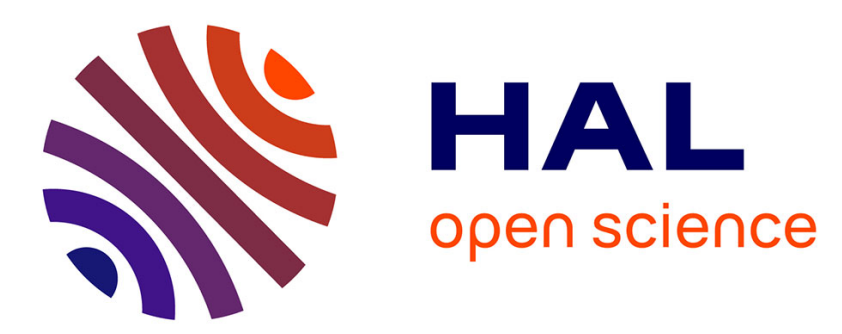

\title{
Optimal displacement of a mobile telemedicine unit in an urban disaster scenario
}

John Puentes, Luc Pigeon, René Proulx, Alexandre Bergeron-Guyard, David Belanger

\section{- To cite this version:}

John Puentes, Luc Pigeon, René Proulx, Alexandre Bergeron-Guyard, David Belanger. Optimal displacement of a mobile telemedicine unit in an urban disaster scenario. International Journal of Biomedical Engineering and Technology (IJBET), 2009, 2 (1), pp.44 - 73. 10.1504/IJBET.2009.021907. hal-02166948

\section{HAL Id: hal-02166948 \\ https://hal.science/hal-02166948}

Submitted on 9 Jun 2021

HAL is a multi-disciplinary open access archive for the deposit and dissemination of scientific research documents, whether they are published or not. The documents may come from teaching and research institutions in France or abroad, or from public or private research centers.
L'archive ouverte pluridisciplinaire HAL, est destinée au dépôt et à la diffusion de documents scientifiques de niveau recherche, publiés ou non, émanant des établissements d'enseignement et de recherche français ou étrangers, des laboratoires publics ou privés. 


\title{
OPTIMAL DISPLACEMENT OF A MOBILE TELEMEDICINE UNIT IN AN URBAN DISASTER SCENARIO
}

\author{
J. Puentes ${ }^{1,2}$, L. Pigeon ${ }^{3}$, R. Proulx ${ }^{4}$, A. Bergeron-Guyard ${ }^{3}$, \\ D. Bélanger ${ }^{4}$ \\ ${ }^{1}$ GET-ENST Bretagne, Département Image et Traitement de l’Information, Brest, France \\ ${ }^{2}$ INSERM U650, Laboratoire de Traitement de l’Information Médicale, Brest, France \\ ${ }^{3}$ Defense Research and Development Canada, 2459 Pie-XI North, Quebec City, Canada \\ ${ }^{4}$ Thalès System Canada, Québec, Canada
}

\begin{abstract}
Mobile telemedicine systems could improve disaster scenario medical response, serving to cope partly, with the crucial insufficiency of resources. Mobility is useful to provide essential remotely assisted care to victims where they were found, in addition to evacuate them. Deciding mobile units' intervention adds to the complexity of disaster response coordination. This paper reports on our research work for the development of a geographic aware application, that we propose to use as a decision support tool to optimize mobile unit displacements in dynamic environments. Four simulated scenarios were used to test the system anticipation capacity to face evolving threat conditions (toxic cloud dispersion). Results show how the trajectories are flexibly adjusted according to identified constraints.
\end{abstract}

Keywords: Mobile telemedicine, threats management, disaster scenario, optimal path, graphs. 


\section{Biographical Notes}

John Puentes holds an Electronics Engineering degree, a M.Sc. of Image Processing and Artificial Intelligence, and a Ph.D. in Signal Processing and Telecommunications. He worked as engineer, consultant and project manager for biomedical and telecommunications companies, before moving to the Image and Information Processing department at GET École Nationale Supérieure des Télécommunications de Bretagne, where he is assistant professor and associate researcher of the French Institute of Health and Medical Research. He is an invited associate editor and reviewer for several international journals, conferences and organisations. His primary research interests are medical practice support systems, image indexing, and telemedicine.

Luc Pigeon received the B.Sc. A. and the M.Sc. in Geomatic Engineering from Université Laval (Qc, Canada) in 1995 and 1997 respectively. He received a Ph.D. degree in Electrical Engineering from the École Nationale Supérieure des Télécommunications de Bretagne (France) in 2001. Since September 2000, he is Defense Scientist at the Defense Research and Development Canada (DRDC) research centre in Valcartier (Qc, Canada). He is currently the Scientific Authority for R\&D in Urban Operations - Command and control. He is also Canadian scientific delegate for NATO studies on Urban Operations and for US studies on catastrophic warfare. His main research interests are information fusion and intelligent systems design.

René Proulx is a mathematician and computer scientist. He graduated from Université Laval (Québec) with Bachelor and M. Sc. degrees in Mathematics followed by an M. Sc. degree in Computer Science (Université Laval, Québec). After teaching mathematics for a few years he went into the consulting business working for consulting firms in Québec. He is currently a senior systems analyst for Thales Canada Inc., Systems Division. He has been involved in the scientific community and worked closely with Defense Research and Development Canada (DRDC) on R\&D projects for over 20 years. His research interests include Command, Control, Communications, and Intelligence (C3I) systems, simulation modeling and analysis, evolutionary computations, quantum computing and cryptology.

Alexandre Bergeron-Guyard holds a B.Sc. in Computer Science from the University of Montreal. He is Computer Scientist at the at the Defense Research and Development Canada (DRDC) research centre in Valcartier (Qc, Canada). His current research interests are reinforcement learning and Markov decision process, specifically tailored for path finding in evolving urban environments.

David Bélanger holds a B.Sc.A. in Microelectronics from the Université du Quebec, Montreal (Qc, Canada) and a M.Sc. in Electrical Engineering applied to Artificial Intelligence from the Ecole de Technologie Supérieure (Qc,Canada). Since 2006 he works for Thales System Canada. His main research interests are graph theory and intelligent systems. 


\section{Mobile telemedicine and disaster scenarios}

Disaster scenarios arise when the available resources are largely insufficient to provide an appropriate emergency response as a consequence of the number of victims, within a risky an uncertain environment, provoking diverse social and economic consequences. Most of them are also associated to a significant transportation and communication infrastructure damage. Considering that it is the event effect on the existing emergency intervention capacity, and not necessarily the event scale that will determine this complex situation, a disaster scenario can take place at multiple temporal and spatial extents.

Among the technology-based resources with potential impact on improving the required medical response is telemedicine (Teich et al. 2002, Chan et al. 2004), which may involve the use of mobile systems, depending on the scenario features. Despite the wide interest given to telemedicine, there are few disaster oriented analyses (Pattichis et al. 2002). Known studies report on the use of satellite communications to support rescue and relief efforts, compensating for required additional medical personnel in warfare and earthquake scenarios, through remote consultation with other countries in the most critical medical specializations (Garshnek and Burkle 1999), epidemic follow-up (Diez 2002), and emergency consultation (Feliciani 2003). On the other hand, works on mobile telemedicine systems (Istepanian et al. 2004, Widya et al. 2006, Herscovici et al. 2007) have concentrated on critical system components like the necessary communications infrastructure, the type of transmitted data, and the innovations outcomes. An implicit assumption regarding these systems is that the mobile element (portable unit or equipped vehicle) displacement is either guaranteed without restrictions, or does not require a particular consideration. This is not the case of disaster scenarios or warfare, on which numerous threats could hamper the telemedicine unit displacements, forcing it to avoid danger while moving towards a previously decided place.

A mobile telemedicine response can be carried out in different manners: diagnosis during ambulance transport (Xiao et al. 2000), mobile vehicle for very close to home examination (Takizawa et al. 2001), or wireless portable units (Cabrera et al. 2001, Kyriacou et al. 2003, Tachakra et al. 2003, Chu and Ganz 2004, Rasid and Woodward 2005). The different degrees of mobility and flexibility of these solutions are likely to be appropriate for intervention in several situations. Figure 1 describes a non exhaustive characterization scheme to illustrate some of the possible relations between the context, components and telemedicine responses. For convenience 3 orthogonal axes have been used, but the elements order along each axis is arbitrary and not related to any particular degree of importance. Three contexts can be considered as the most representative - emergency, warfare and natural disaster -, which may be associated to one or more components - industrial accident, flood, heat wave, mudslide, earthquake, epidemic, hurricane, and terror attack-, and would allow one or several types of telemedicine response - fixed unit, equipped ambulance, multi-utility mobile vehicle, or mobile compact units. For any case, a Control and Command Post (CCP) in charge of the medical response management, decides 
when and where the intervention actions, including mobile telemedicine deployment, will take place (Benner et al. 2004). Handling each mission hazards necessitates a coordinated effort focused on specific events (Mackenzie et al. 2005). In this sense, geographic aware applications emerge as a useful tool to support in part those efforts (Cabrera et al. 2001, Teich et al. 2002, Chan et al. 2004). Particularly, in the context of our study, terrestrial navigation assistance appears as a useful alternative to aid the mobile telemedicine unit drivers, optimizing their displacements, according to the scenario evolving conditions.

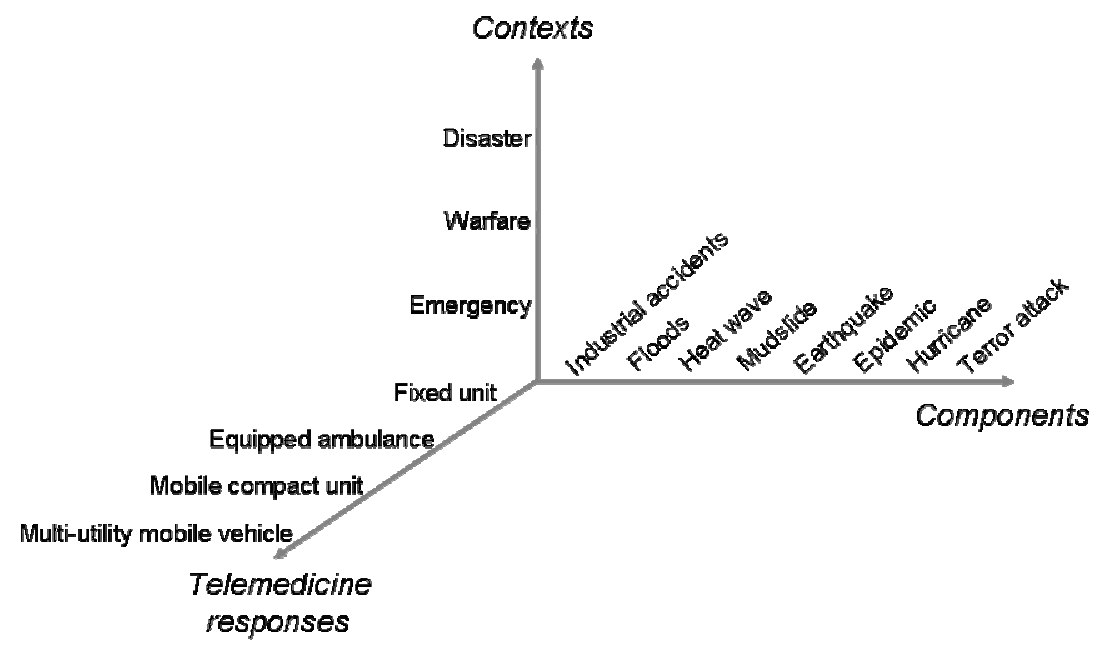

Figure 1 Characterization of context, components and telemedicine responses

Currently, numerous off-the-shelf tools can solve the problem of optimal path determination between two points. They take into account some parameters like: roads speed limits, vehicle fuel consumption, and user constraints as budget and expected travel time, eventually updating information about public works, traffic, and road closures. Representative examples are Garmin (www8.garmin.com/traffic/fm/index.jsp), Google Maps (maps.google.com), MapPoint (www.microsoft.com/mappoint) and MapQuest (www.mapquest.com). These tools are also characterized though, by their inability to function in a dynamic environment (except for traffic flow models), which require forecasting future situations along with their inherent uncertainties. This is the case of a disaster scenario, where only continuous data and knowledge integration give properly adapted navigation support. Our work proposes the validation of such system, capable of being anticipative, instead of reactive, according to the estimated threat accuracy and certainty evaluation, threat tolerance and adaptable emergency level inferences. In the rest of the paper, threat management is presented after a simplified description of the navigation system elements. An experimental validation based on a simulated dynamic disaster scenario examines four possible situations, permitting to appraise the interest of anticipation and threat management, to enable optimal displacement of mobile telemedicine units. 


\section{Navigation system elements}

Before a coordinated medical emergency response is provided in a disaster scenario, the CCP must collect the information about victims' injuries, as well as the status of available qualified personnel (physicians, paramedics) and equipment (ambulances, vehicles, portable telemedicine units), among others. Victims are expected to be identified according to injuries gravity, before being transported either to an advanced medical site or to a properly equipped hospital. Their evacuation is thereafter organized depending on priorities, identified destinations and possible access paths. Otherwise, portable telemedicine units should be transported to the areas where victims have been found. It is at the victim evacuation or unit transportation stage that the proposed system intervenes, to improve real-time resource planning decision support, being the main objective to optimize the displacement of the mobile elements (ambulance, vehicle or compact units), considering the threats estimated impact. To solve this problem the mobile element is constrained to travel on a weighted network, depending on the weights provided by two main knowledge streams: the terrain itself and other agents traveling on the same network. Measured geographical positions and times of a telemedicine unit in motion, in addition to information about surrounding area incidents that could have an effect on the unit displacement, are also introduced on real-time to the navigation system.

This section presents the basic representation framework and model used. It is outside the scope of this paper to review the large number of algorithms and techniques used in path finding and dynamic constrained optimization, reference examples can be found in Stentz 1994, Ramalingam et al. 1996, Korkmaz 2001 and Trovato et al. 2002.

\subsection{Graph representation}

The physical characteristics of the disaster zone terrain constitute the basic navigation network elements, including terrain geometry and transportation infrastructure. Many data models and frameworks exist to represent road networks and related data such as the National Road Network ${ }^{1}$ (NRN), the Geographic Data File ${ }^{2}$ (GDF) and the National Spatial Data Infrastructure ${ }^{3}$ (NSDI). However, our application integrates many static or dynamic entities which are not necessarily associated to roads (e.g., threats, capabilities) and require a different model for their attributes (see section 2.3). In our context, for road data we use the natural mapping of a road network to a directed graph, transforming a navigation problem on a road network, to a spatial graph theoretic problem. A directed graph $\vec{G}$ is usually defined as $\vec{G}=(V, \vec{E})$ where $\mathrm{V}$ is a set of vertices (or nodes), and $\vec{E}$ is a set of directed edges (or arcs) joining two vertices, or the same vertex. In our case, the graph represents a road network,

\footnotetext{
${ }^{1}$ http://www.geobase.ca/geobase/en/data/nrn/index.html;jsessionid=0815B100214694A7 D949ACB7EE63E7D3

${ }^{2}$ http://www.ertico.com/en/links/links/gdf_-

geographic_data_files.htm\#GDF_3.0_Documentation_\&_Manual

${ }^{3}$ http://www.fgdc.gov/nsdi/nsdi.html
} 
where vertices symbolize streets intersections, and edges stand for the streets segments between adjacent intersections and their direction. Table 1 shows examples of the amount of vertices and edges for different cities, stored in the system graphs database.

Table 1 Graph elements for different cities

\begin{tabular}{cccc}
\hline City & Surface $\left.\mathbf{( k m}^{\mathbf{2}}\right)$ & Vertices & Edges \\
\hline Boston & 232 & 56.145 & 152.357 \\
Quebec City & 542 & 15.417 & 38.867 \\
San Diego & 963 & 33.376 & 91.376 \\
Seattle & 369 & 67.079 & 170.386 \\
Vancouver & 114 & 52.705 & 139.406 \\
Washington DC & 176 & 39.616 & 110.200 \\
\hline
\end{tabular}

A number of attributes ${ }^{4}$ are also associated to each vertex and edge such as: Vertex:

- vertex id (intersection street names); Edge:

- type of intersection (traffic light, stop, etc.);

- edge id (source and end vertex i.e., driving direction between two adjacent intersections);

- range of civic numbers;

- one-way or not;

- geographic orientation;

- road type;

- maximum vehicle speed allowed;

- maximum vehicle height allowed;

- width or number of lanes;

- traffic or control signs;

- length.

Environment and scenario conditions evolve constantly, introducing dynamic constraints and changes to the initial graph that becomes dynamic by the addition or suppression of vertices and edges, as well as updates of their attributes values, on a permanent basis. Hence, the path research algorithm used to guide the mobile telemedicine unit in the urban disaster scenario is adapted accordingly.

\subsection{Cost function}

In order to guide a mobile unit from a start to an end point it is necessary to define a path, allowing continuous displacement through a coherent and directed sequence of edges. At its simplest expression, a path $\mu$ of length $q$, is a sequence of $q$ edges:

$$
\mu=\left\{\vec{a}_{1}, \vec{a}_{2}, \ldots, \vec{a}_{q}\right\}
$$

The edges lengths $l\left(\vec{a}_{i}\right)$ associated to a given path, can be summed to obtain the

\footnotetext{
${ }^{4}$ Some of the attributes listed are not always present in the source data files or layers of information available.
} 
path cost $l(\mu)$ :

$$
l(\mu)=\sum_{i} l\left(\vec{a}_{i}\right)
$$

Besides a length-based path cost, other associated values (road conditions, risk, weather, etc.) can be combined and processed as a vector to define an edge weight function $w\left(\vec{a}_{i}\right)$, and the resulting path cost $w(\mu)$ :

$$
w(\mu)=\sum_{i} w\left(\vec{a}_{i}\right)
$$

Some components of the weight function are dynamic, depending on time and other factors (e.g., capabilities). Hence, the weight of an edge cannot be established in advance except for simple cases where we consider only the edge length or other invariant edge attribute. As a consequence, each edge weight is evaluated during computations, according to the particular values of its dynamic components.

Commonly, there will be many paths between the start and the end point. The optimal path is defined by the minimum cost with respect to the appraised constraints and used cost function.

The system generalizes cost functions specifying them as a linear combination of smaller constituent cost functions including, among others:

- $C_{1}$ : threat cost along path (fragmented into several costs for different types of threat e.g., obstacles, potentially harmful element, toxic deposits, expected casualties, etc.);

- $C_{2}$ : total path distance cost;

- $C_{3}$ : total travel time cost along path;

- $C_{4}$ : total cost of operation;

All these functions depend upon the particular segments which compose the path and may vary also according to time, vehicle characteristics or capabilities, and other variables (e.g., emergency behavior and threat tolerance degrees). This yields a resulting overall cost function:

$$
C(p)=\sum_{i=1}^{k} \alpha_{i} C_{i}(p)
$$

where $\alpha_{i}$ is the linear combination coefficient for function $C$, and $C_{i}(p)$ is the cost function for path $p$. The cost function parameters can be adjusted according to a particular navigation scenario or objective.

\section{$2.3 \quad$ Complementary modules}

Intervention personnel already deployed in the area, who are moving on the same network as the guided mobile element, send information to the CCP, for instance threat identification, using VHF band transceivers capable of functioning independently of public communication networks. On the other hand, weather information (temperature, precipitation, air pressure, humidity, wind force and direction, etc.), police and other intervention staff positions in the disaster zone, along with hospitals congestion levels, is read from distant servers. Supplementary information can also be obtained in the form of satellite or aerial 
images, and residents reports. Information from voice communications or hardcopy must be manually entered in the system while automated information sources are fed into the system through network connections or Web Services access. These data are processed by means of modules like source characterization (credibility and reliability), accuracy assessment (spatial and temporal), threat edition, threat estimated progression, information fusion, graph weights adjustment, and prior knowledge extrapolation.

The underlying model of the system is based on NATO's Joint Command, Control and Consultation Information Exchange Data Model JC3IEDM (NATO 2005) which allows modeling of all entities evolving in the theater of operations e.g., scenarios, vehicles, capabilities, threat and toxic material characteristics, geographic features, routes, weather conditions, information reports etc. A Geographical Information System (GIS) engine (LuciadMap) is embedded in the system and used for the visualization of the theater of operations and its evolving entities. Source data for the road networks can be imported as ESRI shapefiles or VMAP vector maps and the application allows connection to Open Geospatial Consortium WMS (Web Map Service) and WFS (Web Feature Service) servers so that additional layers of information can be added to the display from the application. Conversely, the application includes a WMS server that allows external system to import paths computed by the application. Data persistence in the system is maintained in an Oracle Spatial database, consisting mainly in the model instance data and the graph data that represent the source road network data.

\section{$2.4 \quad$ User interface}

A user interface allows configuring varied scenarios, which include all the information about the field threats, the mobile element and the scenario management tools. Data required at this stage are: the vehicle type (automobile, ambulance, or truck); the type of searched path (quickest or shortest); the threat tolerance (between safest and hazardous); the threat responsiveness (anticipative or adaptive); the start and end point geographical coordinates obtained by clicking directly on the zone map; the start time; and the emergency behavior (between normal and very urgent). Thereafter, the user requests a path which is immediately displayed over the map. A specific segment of the path can be selected to edit traffic data corresponding to the current time, and ask for a new path. When threats are reported, the area where they are occurring is selected with a zoom in, and the type(s) indicated. The system considers those alterations providing a modified path that takes into account all the previously stated constraints. Several scenarios can be displayed simultaneously thus allowing to view multiple paths from/to different starting/ending points. Depending on the scenarios parameters, these could represent alternative paths for a single vehicle or individual paths for multiple vehicles. Paths, obstacles, threats and other entities are represented on different layers of the map display and the user may select the layers he/she wishes to visualize at any given time. 


\section{Threat management}

Beyond the interpretation of dense traffic as an obstacle to achieve the planned telemedicine mobile element displacement on time, a threat implies that lack of attention to an impending danger could result in the impossibility of accomplishing the displacement, the lost of valuable resources (personnel and material) and the increase of casualties. Our system takes into account several types of threat and manages them according to information updates. System response to information updates depends upon their impact on currently planned travel routes for the mobile element and on the selected planning strategy (i.e., anticipative or adaptive) as described hereafter.

\subsection{Threat modeling}

Threats are generated by the occurrence of specific time-stamped events recorded in the system's database. Each of such events is associated to specific information:

- time of occurrence: time at which the event was observed or detected;

- event location: geographical location or area at which the event was observed/detected or spatial reference point for the event;

- resource(s): material, facility or organization which caused the event;

- capabilities: resource characteristics enabling it to produce effects;

- effect(s): possible or expected consequences of the threat event resource capabilities.

Four types of threat events are currently supported by our system:

1. Presence of a potentially harmful element.

2. Presence of a road obstacle (e.g., road block).

3. Occurrence of a Chemical, Bacteriological, Radiological, Nuclear or high-yield Explosive (CBRNE) event.

4. Specification of an area to avoid.

Threats generated by such events may be static over a fixed location/area or dynamic (i.e., threats whose characteristics and effects may vary continuously or discreetly in time and space). Table 2 shows some examples of threats that can be modeled within this framework (JC3IEDM data model).

\subsection{Threat updates}

Information updates on threat events (as well as other types of information or complementary data relevant to the situation) may come from various sources, such as: the telemedicine mobile unit itself, other rescue personnel deployed in the disaster area, imagery or electronic devices and sensors. In some cases, the information may be provided by an external system and include situation predictions based on prior knowledge to which prediction models are applied (e.g., toxic cloud dispersion prediction). For example, a moving toxic cloud threat is modeled using an atmospheric transport model for turbulence, a massconsistent wind field model for flow over terrain and/or an urban dispersion model. Many systems (see Section 4) that provide hazard cloud dispersion information use a Gaussian puff second order closure model (e.g., SCIPUFF) for turbulence and models derived from terrain and tunnel experiments (e.g., SWIFT, UDM). 
Table 2 Threat event examples and their characteristics

\begin{tabular}{|c|c|c|c|c|c|}
\hline Event & Resource & Capabilities & Threat & Effects & $\begin{array}{c}\text { Static- } \\
\text { Dynamic }\end{array}$ \\
\hline $\begin{array}{c}\text { Potentially } \\
\text { harmful element } \\
\text { detected/ } \\
\text { encountered }\end{array}$ & $\begin{array}{l}\text { Potentially } \\
\text { harmful } \\
\text { element }\end{array}$ & $\begin{array}{l}\text { Potentially harmful } \\
\text { element type and } \\
\text { capabilities }\end{array}$ & $\begin{array}{c}\text { Potential } \\
\text { engagement }\end{array}$ & $\begin{array}{c}\text { Possible } \\
\text { damage/casualties } \\
\text { to own } \\
\text { vehicle/crew }\end{array}$ & $\begin{array}{c}\text { Static/ } \\
\text { Dynamic }\end{array}$ \\
\hline Obstacle & Road block & $\begin{array}{l}\text { Material and } \\
\text { dimensions }\end{array}$ & $\begin{array}{c}\text { Path access } \\
\text { denial }\end{array}$ & $\begin{array}{l}\text { Road segment } \\
\text { blocked may not } \\
\text { be used in a path } \\
\text { (unless vehicle has } \\
\text { the capability to } \\
\text { overcome } \\
\text { obstacle) }\end{array}$ & Static \\
\hline $\begin{array}{c}\text { Industrial } \\
\text { chemical } \\
\text { incident } \\
\text { (CBRNE) event }\end{array}$ & Toxic cloud & $\begin{array}{c}\text { Gas identification } \\
\text { and characteristics } \\
\text { (symptoms vs. } \\
\text { dosage) }\end{array}$ & Contamination & $\begin{array}{l}\text { Possible casualties } \\
\text { to vehicle crew, } \\
\text { toxic deposits on } \\
\text { vehicle and } \\
\text { contaminated area }\end{array}$ & Dynamic \\
\hline Flood & Water & $\begin{array}{l}\text { Surface of flooded } \\
\text { area, depth of } \\
\text { water }\end{array}$ & Access denial & $\begin{array}{l}\text { Path may not go } \\
\text { through flooded } \\
\text { area (unless } \\
\text { vehicle has } \\
\text { sufficient } \\
\text { amphibious or } \\
\text { depth clearance } \\
\text { capabilities) } \\
\end{array}$ & $\begin{array}{c}\text { Static/ } \\
\text { Dynamic }\end{array}$ \\
\hline
\end{tabular}

The system behavior with respect to threat information updates allows two types of response: adaptive or anticipative.

In adaptive mode, the system considers only factual ${ }^{5}$ information about the status of known threats. If the unit is already moving along a previously planned path, current locations and areas affected by the known threats are checked against the remaining portion of the path for intersection, in which case this portion of the path is recomputed to avoid the threats according to the cost function optimization criteria. If a new path is to be planned for a unit, then the path is computed from start to destination locations in the same way. This

\footnotetext{
${ }^{5}$ One should be careful about the interpretation of "factual" in this context. Factual information does not necessarily mean ground-truth information, as it is always subject to accuracy and source reliability or credibility. For our purposes, factual information represents information that is known by the system about the current situation. Information that the system may know about the situation in the future i.e., predictions, is not considered factual in this context.
} 
strategy can be viewed as taking a snapshot of the situation at the current time and planning (or readjusting plan) as if the situation would remain fixed. Consequently, any predictive information will be disregarded by the plan. Actually, predictive information may be present in the system database but in adaptive mode the system tries to optimize its solution path as if the predictions will later prove to be wrong, or contradicted by new information and removed from the database.

In anticipative mode, the system takes also into account predictive information i.e., the current and future locations of the mobile unit and the current and future statuses of known and predicted threats. If the unit is already moving along a previously planned path, current locations and areas affected by the known threats are checked against the current location of the unit. Future locations and areas affected by the predicted threats are checked against corresponding estimated future locations of the unit on the remaining portion of the path. Whenever intersection occurs, the corresponding portion of the path is planned again in time to avoid the threats according to the cost function optimization criteria. If a new path is to be planned for a unit, then the path is computed from start to destination locations, according to time synchronized estimated/predicted locations of unit/threats in the same way. This strategy can be viewed as looking ahead in time, or simulating the situation evolution to see if the unit will encounter a threat later in time according to the path previously (or currently) being computed and the predictions on threats behavior. Consequently, the system will use any predictive information it can infer (e.g., estimate threat progression) or has obtained to optimize the plan. Note that this mode does not rule out instantaneous adaptive behavior upon irregular inputs (e.g., sudden encounter of a previously unreported threat).

Whether the selected strategy should be adaptive or anticipative is a matter of information confidence, reliability and accuracy. It also depends on how safe or cautious one wants to be (though this aspect can also be captured by the cost function parameterization). Predictions have an inherent uncertainty which adds up to the inevitable imprecision of factual information measurements. Taking predictions into account can yield to the selection of a path which may be shown later to be far from optimal, if the prediction proves wrong. On the other hand, not considering predictions can force costly path readjustments later, which would make an otherwise optimal initial path transform into a very bad one, whilst the predictions could have suggested a rather good path from the beginning. On a more conceptual level, assuming stationary state as in the adaptive mode is also a form of prediction, albeit perhaps not a good one unless one has strong reasons to do so, which brings us back to the importance of confidence and reliability of information.

From the user interface point of view, the adaptive vs. anticipative views present a difficulty in displaying a meaningful image of the situation on the GIS. This difficulty is even more important since the system may be used to display paths that are planned in the future and/or monitor previously planned paths that are already in execution. To distinguish both, a different color is used to display 
paths (or portions of paths) which are not yet completed from those which are currently executing and being monitored. Thus, for a path currently in execution, vehicle position reports will gradually alter the color of the path displayed and show the vehicle progress along the path. For the display of obstacles or threats, the predictions are displayed only when monitoring a plan in execution and at the time of the prediction. However, when a plan is made for a vehicle in anticipative mode, the resulting path computed takes into account the future menace though it will not appear on the map where the planned path is displayed. This is not an ideal situation since a planned path may appear to make unnecessary detours on the map only because there are forecasted but not displayed threats that force such detours. Displaying the forecasted threats would not solve this problem, given that parts of the path might appear to go right across a threat, considering that the expected times of passage of the vehicle and of the threat are not synchronized, and there is actually no expected danger. The whole problem of correctly representing mixed time and space events on a single image display is by no means a simple one. In future releases of the application, we intend to develop a partial solution that would enable the use of a time slider control to support look ahead and backwards displays of the current situation on the map in a movie-like fashion.

\section{Knowledge base}

The system requires several input sources to process and construct a coherent picture of the current situation and to make inferences that allow foreseeing its evolution. The completeness of this picture and the accuracy of its predicted evolution have a direct impact on the quality of the path solution that is computed. To achieve timely awareness of the situation, interoperability between system components and between these with external systems is required. The core component of the system is responsible for building, maintaining and providing the knowledge required by the path optimization component and other system components as well. Figure 2 illustrates the structure and relations between the system knowledge base repository, the system main components and main external knowledge system providers.

The Central Intelligence Component (CIC) is the system core knowledge integration and management component. Knowledge from external sources is provided to the CIC according to synchronous/asynchronous subscriber or query protocols. For example, we use the GlobalWeather ${ }^{6}$ Web Service to provide updates on weather conditions. The system automatically sends service requests for weather updates every five minutes and new forecasts are integrated in the system's database. The connection parameters can be changed to connect to other servers or use different refresh rates. In a similar way, CBRNE toxic cloud dispersion model calculations and hazard predictions are performed by external systems namely, the Sensor Analysis and Fusion Environment (SAFE) developed by the United Kingdom Defence Science and Technology Laboratory (Bull 2004) and the Hazard Prediction and Assessment Capability (HPAC) developed by the

\footnotetext{
${ }^{6}$ http://www.webservicex.com/globalweather.asmx
} 
U.S. Defense Threat Reduction Agency (DTRA 2005) ${ }^{7}$. Other dispersion prediction systems exist such as the Area Location of Hazardous Atmospheres (ALOHA) and the Computer-Aided Management of Emergency Operations (CAMEO), developed jointly by the U.S. National Oceanic and Atmospheric Administration (NOAA) and the U.S. Environment and Protection Agency (EPA). However, none of these models has any capability to model radiological scenarios, as they cannot assess radiation doses and deposits over contaminated areas. The U.S. National Atmospheric Release Advisory Center (NARAC) ${ }^{8}$ also offers online expert services and tools (e.g., NARAC IClient, NARAC Web) for the assessment and modeling of hazard material release and dispersion which include radiological and nuclear incidents but these services and tools require access authorizations restricted to specific organizations.

System Knowledge Sources

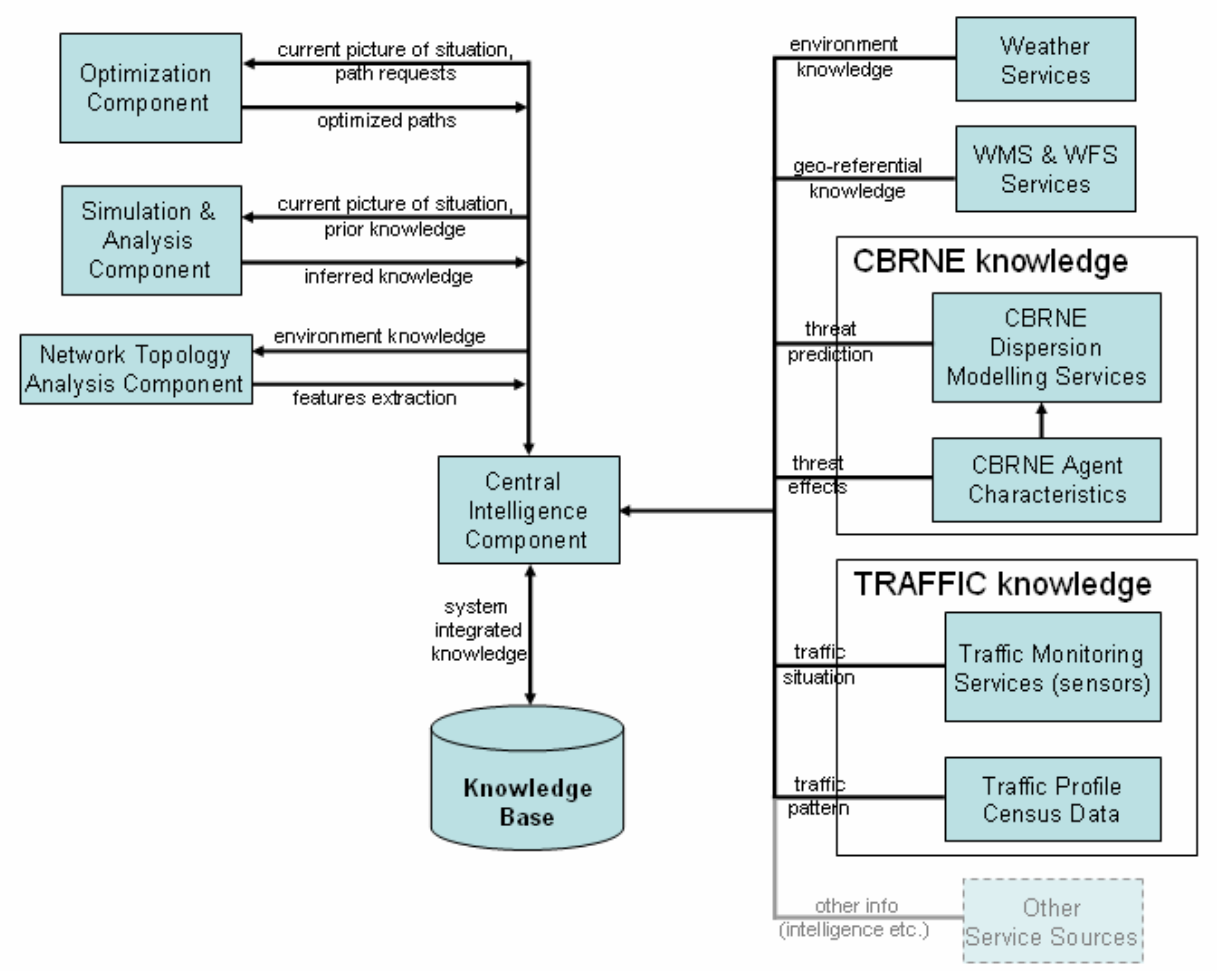

Figure 2 System knowledge management

Another example of the application using external knowledge source is for the collection of traffic information. Two different kinds of knowledge are used: a

\footnotetext{
${ }^{7}$ These systems can be used locally with raw estimation or simulated data for initial approximations but require downloaded numerical weather prediction files (HPAC and SAFE) or sensor data observations (SAFE) from external systems for maximum accuracy.

${ }^{8}$ https://narac.llnl.gov/
} 
priori data and real-time data. The first one is used in absence of or complementary to the second. A priori data are generated offline, using sensor measurements, surveys of transport habits of the population and different techniques of macro-simulation ${ }^{9}$ and stored in public access databases. These data are tallied over large periods of time (usually yearly) and provide a typical profile of average traffic flow up to a certain granularity. Such profiles are usually compiled according to days and time periods within the days. They can be used as an approximation of usual traffic conditions when no incident (accident, bad weather condition, special attraction, etc.) are present on the road network. Of course, such incidents do happen and it is why our application can also access real-time information on traffic from Web Service providers ${ }^{10}$, in the same way that it receives weather information. In the case where traffic profiles and traffic Web Services do not exist or are unavailable for the area of operations, the system can still use basic information on speed limits (generally available in the map shape files). The system also allows the user to select road segments or areas directly on the GIS display using different filters e.g., direction, road type, and to directly modify or enter an estimation of the traffic flow on the segments according to whatever information he/she may have. Within the system the traffic flow, speed limits, maximum speed of the vehicle and weather conditions affecting traffic are processed to produce an estimation of the maximum achievable speed (MAS) for the vehicle on each road segment. The MAS values are the baseline reference for the emergency behavior of the vehicle. For example, when the behavior is normal, the vehicle will be considered to move at a speed similar to the speed of the traffic flow unless that speed is higher than the MAS for each traveled segment. If the behavior corresponds to a very urgent situation, the vehicle will be considered to assume MAS values along each segment. Other emergency behaviors between normal and very urgent yield proportional estimated vehicle speed between these two limits.

Since the system relies on a number of external sources of knowledge it is dependent on the performance of these systems. For example, gas dispersion estimation prediction models can take several minutes of computer processing time, depending on the size of the affected area, accuracy and time horizon of the results and complexity of the model. In addition, the external systems may not be accessible due to communication failure or incapacitated external server.

To account for the possibility that communications with an external source may not be available or that processing time from the service source provider may be too long, the system provides the operator with the possibility to enter quick estimations in the system or use default values. The user interface includes graphical tools to manually input traffic levels of congestion and average/maximum achievable travel speed over road segments or areas, weather conditions, road obstacles, and threat events information. For instance, a chemical release incident may require immediate re-routing of a nearby vehicle

\footnotetext{
${ }^{9}$ http://www.mtq.gouv.qc.ca/portal/page/portal/ministere_en/ministere/recherche_innovat ion transport

${ }^{10} \mathrm{http}: / /$ developer.yahoo.com/traffic/rest/V1/index.html
} 
without waiting for estimates of dispersion from time consuming dispersion models. In such a case, the system can provide and display an immediate ATP-45 (NATO 1987) hazard warning area with a minimum of input information from the operator (i.e., basic weather conditions, source and quantity of release rough estimates). An updated route avoiding or escaping the warning area is computed and displayed at once and provides a temporarily solution while waiting for more precise forecasts of dispersion from another source that is currently unavailable or processing.

System knowledge sources may operate offline (Optimization, Simulation \& Analysis and Network Topology Analysis components) or online (Simulation \& Analysis and Optimization components). The main task of the CIC is to compile and update the picture of the current situation from all available sources (usually the external knowledge sources and the Optimization component) and to integrate persistent knowledge provided from both external and system knowledge sources.

The Optimization component is the component that receives path request parameters and actually performs the computation of the optimal path. As such, it requires information on the current situation and knowledge stored in the system knowledge base which is provided by the CIC. The Optimization component has access to a pool of algorithms, each of which is designed to solve a particular path finding problem instance in a particular environment. It also includes a meta-control layer that determines which algorithm and algorithm parameterization to select according to a number of factors (features and characteristics of the road network, CCP decisions, constraints, etc.).

The Simulation \& Analysis component is designed to find actions that can modify the situation so that the mobile unit could perform its tasks more easily (e.g., setting up road blocks to secure access for emergency unit). For that, it simulates actions to find positive outcomes on the operation theatre. It is also used to simulate "What-if" scenarios that are useful to generate alternative paths. For example: Which path should I take if the wind changes so that the toxic cloud goes south instead of east?

The Network Topology Analysis component uses geo-referential knowledge to analyze, detect and extract topological features of the road network graph. Metrics and features are obtained through clustering, partitioning, graph segmentation and other graph manipulation tools and techniques, providing ways to increase computational performance of the algorithms used by the Optimization component. This component is useful since it allows offline preprocessing computations on large network graphs (over 100,000 nodes/edges) from network map data which are too costly to execute on the fly. The static metrics and topological features thus extracted are added to the knowledge base and can be later exploited by the meta-control layer when path requests are processed. For example, path finding algorithms performance vary according to graph characteristics and metrics such as graph sparsity (Eppstein et al. 1997), vertex/edge connectivity, maximum edge length, etc. In the same way, graph or sub-graph clustering features may be used to greatly reduce the search space by 
partitioning it into smaller components weakly connected. This knowledge is used at the meta-control level to select the algorithm and parameterization that will be applied for a specific network graph in order to maximize efficiency.

\section{Optimal path calculation}

In our context, the optimality of a computed path cannot be ascertained clearly as in simpler cases such as static graphs. In particular:

- source data that may be used in the evaluation of the cost function is subjected to imperfection and uncertainty (e.g., expected symptoms or casualties following exposure to certain dosages of toxic agent);

- continuous variables used in the optimization process are approximated by discretized and interpolated values e.g., toxic cloud predictions are discretized in time but their impact on path segments might be required at any time value (estimated time of passage of the mobile unit on the segment) which calls for interpolation between the closest predictions in time);

- dynamic constraints are considered;

- incomplete and not perfectly accurate set of constraints (e.g., traffic constraint);

- no wait time considered (path planning does not include scheduling of voluntary stops along the path to wait for a threat to go away).

As a result, the proposed path solution is guaranteed to be optimal if the mobile unit departs at the planned start time and passes each intersection along the path at the expected time according to the plan. This condition is seldom, if ever, satisfied in the real world. Hence, the exact optimal path solution is likely to be always unfeasible and we must aim for nearly optimal feasible path solution. To avoid constant re-planning for optimality once the planned path is in execution (i.e., when the vehicle has started to travel along the path), time windows for vehicle passage at each intersection are considered and re-planning is performed only if the time discrepancy between planned and actual times of passage at any intersection along the path exceeds a certain threshold. This requirement means to access the vehicle location e.g., manually operated or automated GPS fixed transmitter device, voice communication, etc. Adding time window tolerances affects the optimality of the feasible path solution. In simulation or when actually monitoring real path execution, parameters must be set for threshold values that will be applied to determine whether the time discrepancy is acceptable or not. Time window tolerances can be further refined using learning techniques such as neural networks provided a sufficient number of training data sets are available (Ahn et al. 2002). Evolutionary computing (e.g., genetic algorithms, simulated annealing) and hierarchical clustering techniques can also be used to find near optimal parameter values or near optimal solutions in path finding and routing problems when the search space (graph) is very large (Botea et al. 2004, Chakroborty and Wivedi 2002).

Apart from time discrepancies between planned and actual times of passage at intersections for a path, there are other factors that could affect the actual path in 
execution optimality. New incoming information reports on events that incur changes in the current situation will trigger recalculation of the path. The events that will trigger path recalculation are:

- changes to a threat, including changes to a threat prediction, new threat occurrence or removal of a threat (applies to all four types of supported threats);

- changes to the traffic conditions;

- and changes in weather conditions (since they may impair the vehicle capabilities along segments affected by the weather change).

Regardless of the condition that required interrupting and re-planning of a path in execution, recalculation of the path is performed by taking the current vehicle location as the new starting point while keeping the same destination.

Changes in the current situation do not only affect path(s) in execution, but also all paths currently planned but not yet started, since the changes may include threat predictions that could interfere with paths planned within the same timespace frame as the predictions. The actual interaction between path (planned or in execution) and update calculations, is implemented by taking advantage of a multi-agent system architecture.

When a new threat is created or modified, its effects are evaluated using threat analyzer agents. The result is a list of affected edges in the road network graph with values representing the quantity of the effect (e.g., dose, flood height, etc.) on each edge. This effect is valid at a certain extent in time and takes the form of an information report of the current situation. Consequently, it is possible to have many reports that represent the same effect at different moments in time (including in the future). Each time a new report is created, an agent checks if the current existing paths (planned or in execution) can be affected by the new threat. If so, the agent sends for each affected path a request to the Optimization component for a new path that will replace the affected one. When the Optimization component computes it, the newly reported information is taken into account, and a path that avoids the threat is found, if necessary. It should be noted that the path request could include new constraints and a different cost function to optimize, than the one previously used to compute the affected path. New information in the current situation and updated paths are always saved in the database and, if required, broadcasted to the clients according to the particular system configuration used as described in the next section.

\section{System implementation and configuration}

The system is currently implemented and tested on PC and Mac platforms running respectively Windows and Tiger operating systems. Since it has been developed in Java and runs within a virtual machine it is therefore portable to UNIX/Linux systems. However, availability and differences in versions of third party applications used by the system (i.e., Oracle, Jini Technology, Toplink and Tomcat) might require some adaptation when porting it to other operation systems. 
The system is implemented according to a client-server architecture. The usual deployment configuration is to have the server application and database management system running on a dedicated base server machine at the CCP location and client applications connecting to it from remote platforms that can be located anywhere provided they support a TCP/IP communication protocol for Internet connection access (local network, wireless, VHF). It can also be configured in a complete local or stand-alone mode with the server, client and database management applications all hosted and running on the same platform. This is of major importance since it allows installing a full system, not just the client part, on a laptop or notebook that can be carried in the vehicle. In this way, should communications with the base server be broken, the system can still be used with its own server and database in a totally autonomous way. Once communications resume, databases are synchronized on both sides. We also have tested the system on palmtops. In this case, only the client application is installed on the palmtop because a full system is yet too demanding in resources for a palmtop. This requires communications with the server to be maintained. Next generations of palmtop devices should allow supporting a full local system in the near future.

The system can also be used even if neither the client application nor the server application is running on the client platform. All that is required is to have an Internet access connection and a Web browser. The system provides Web Services that can be requested by any device connected to the Internet with browsing capabilities. Information and path requests can be input in the system and results are displayed on a Google Map Web page displayed on the device. The Web client also supports on-demand and automatic queries to the server for updates of the current situation. A cell phone with Internet connection and browser could thus be used to access the system. Some functionalities of the system are limited or not provided as Web Services and require the client application (e.g., import/export WMS/WFS), but hazard and obstacles can be reported using only the Web client.

\section{Experimental validation results}

To demonstrate the feasibility and the validity of the proposed threat management approach, four simulated scenarios have been examined using different cities and zones of operations. In all of them, the appropriateness of the recommended optimum path is qualitatively and quantitatively determined. The most complex threat, a toxic cloud in the form of a moving area, is used to simulate an urban disaster. The requested optimal paths simulate a telemedicine mobile unit displacement to a specific point where several victims need medical assistance, or conversely, a situation requiring to transport victims from an incident location to an advanced basic relief station or to a properly equipped hospital. All scenarios use as vehicle an ambulance with telemedicine unit. Simulated scenarios illustrate situations where it is required to:

1. Find a path from a specified medical facility to an incident location.

2. Find paths from an incident location to two different medical facilities. 
3. Find paths from multiple medical facilities to an incident location.

4. Find paths from an incident location to medical facilities with different capacities and waiting times.

In the illustrated examples, a priori averaged traffic data is available from the system database and weather data is provided by an external Web Service source that is queried at regular intervals by the system. The weather data is used by the dispersion model to provide predictions on the evolution of a toxic gas threat.

7.1 Scenario 1 - Dispatching a telemedicine unit to where victims have been found

Assuming that the telemedicine unit is requested to go to a point where a group of several victims have been found by reconnaissance personnel, a first optimal path is given between a hospital (starting point - $S$ flag) and the specific point (end point - $E$ flag). Figure 3 illustrates the resulting path in the absence of any information about eventual threats or hazard areas, but taking into account other information available such as weather and traffic flow. The path displayed is the same regardless of the vehicle behavior mode selected (adaptive or anticipative).

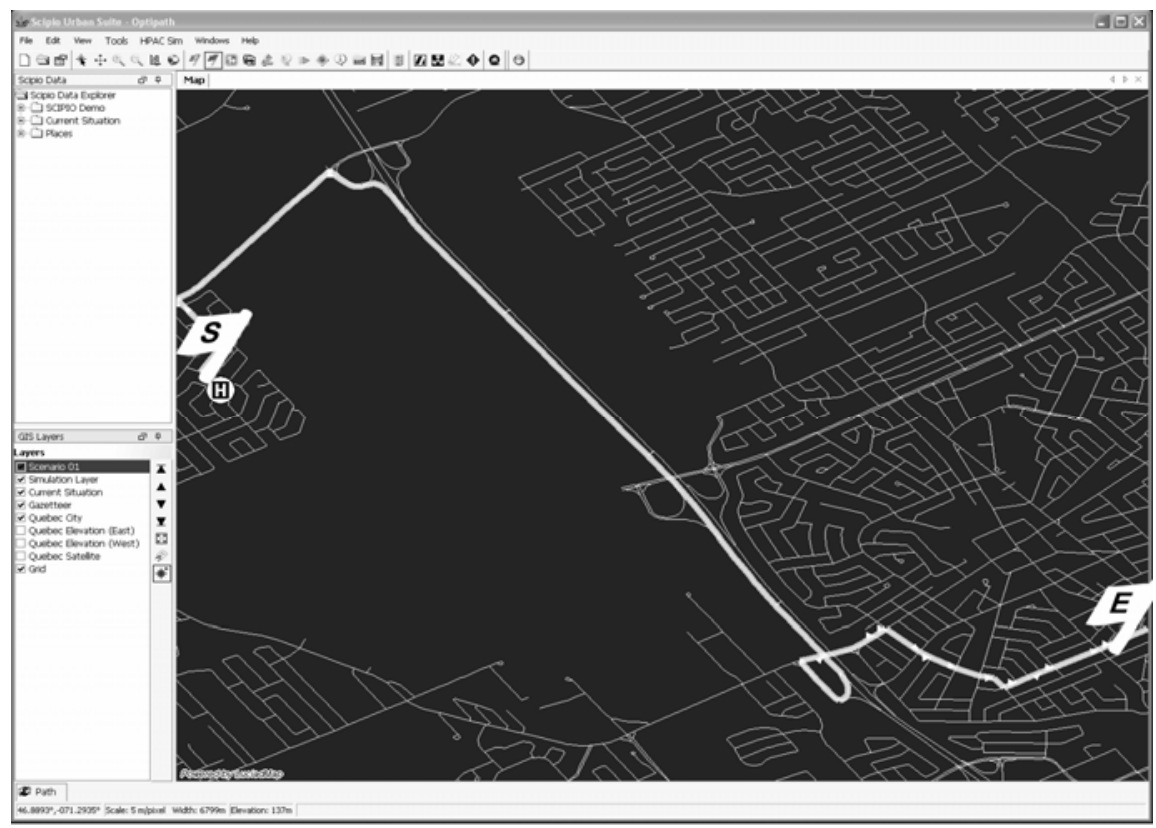

Figure 3 Initial proposed path - No threat information

The next two figures show the same situation when information about a threat is present in the system at the time where the path is requested. A toxic gas release has been observed and two reports about the resulting toxic cloud have been received in the system database prior to the request. The first report contains information on the current situation of the toxic cloud and the second contains a prediction of how the cloud will be dispersed in a given near future according to some dispersion model estimations (e.g., HPAC). Figure 4 shows the system 
response when the behavior is set to adaptive while Figure 5 shows the system response when the behavior is set to anticipative. In both figures, only the current toxic cloud situation (factual information) is displayed, even if predictive information is also accessible in the system. The path computed under adaptive behavior (Figure 4) does not take the available predictive information into account because it assumes that this information may not be reliable or that conditions may change in the meantime. The path for anticipative behavior does take the predictive information into account for the opposite reasons. An area to avoid (seen as an ATP-45 trapezoid warning area enclosing the toxic cloud) indicates how the system depicts the danger zone currently active.

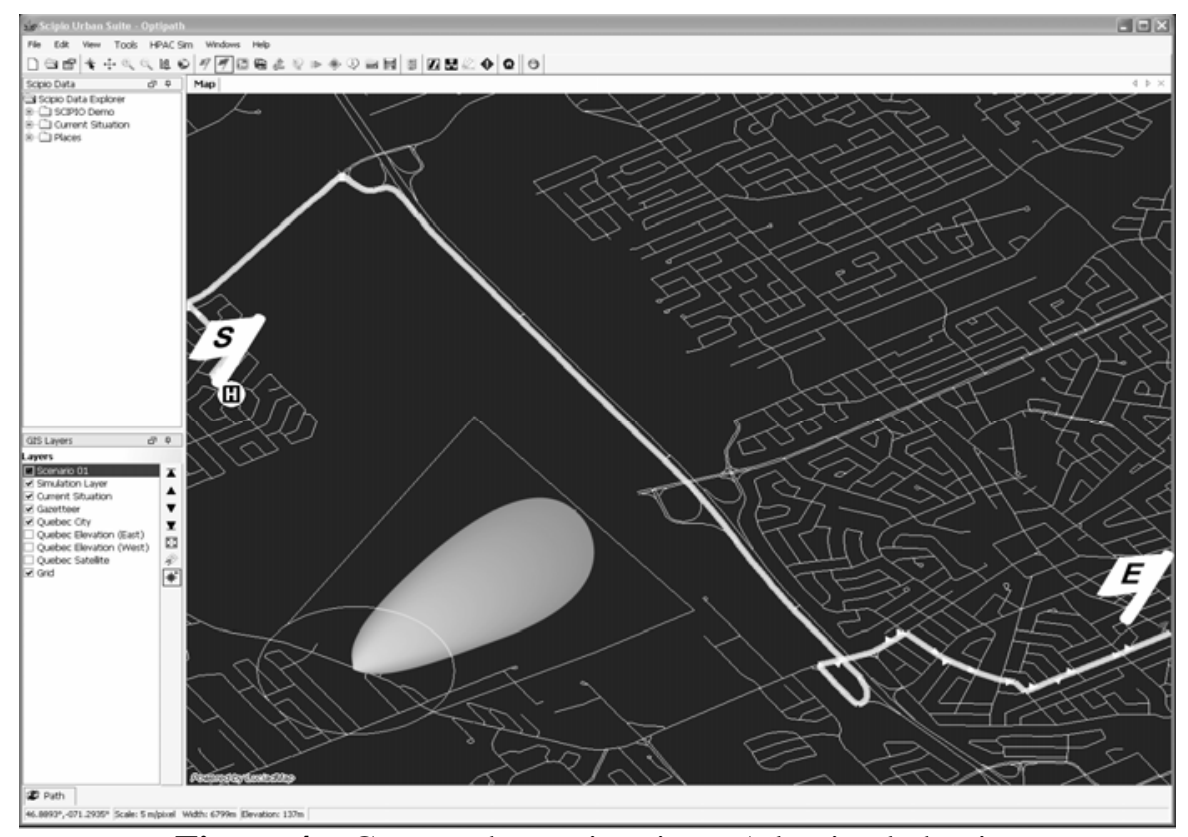

Figure 4 Current threat situation - Adaptive behavior 


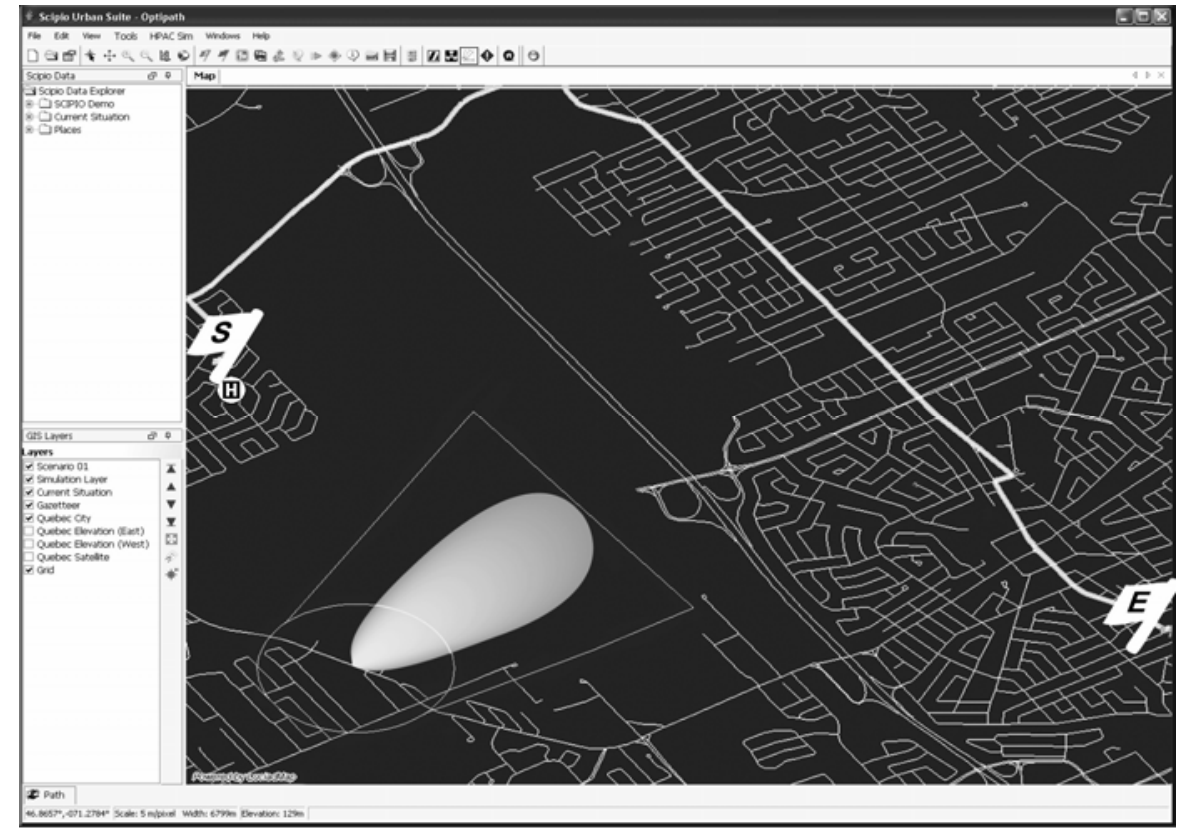

Figure 5 Current threat situation - Anticipative behavior

As can be seen in Figure 5, selecting the anticipative behavior forces the system to look ahead in time for information about a future situation that might be present in the database and determine whether the future situation could affect the planning of the path. In the illustrated case, the system concludes that the predicted dispersion of the toxic cloud is likely to affect execution of the path and produces a different path than in the case of adaptive behavior (Figure 4). The next two figures show what actually has happened when the vehicle follows the path according to both behavior modes. 


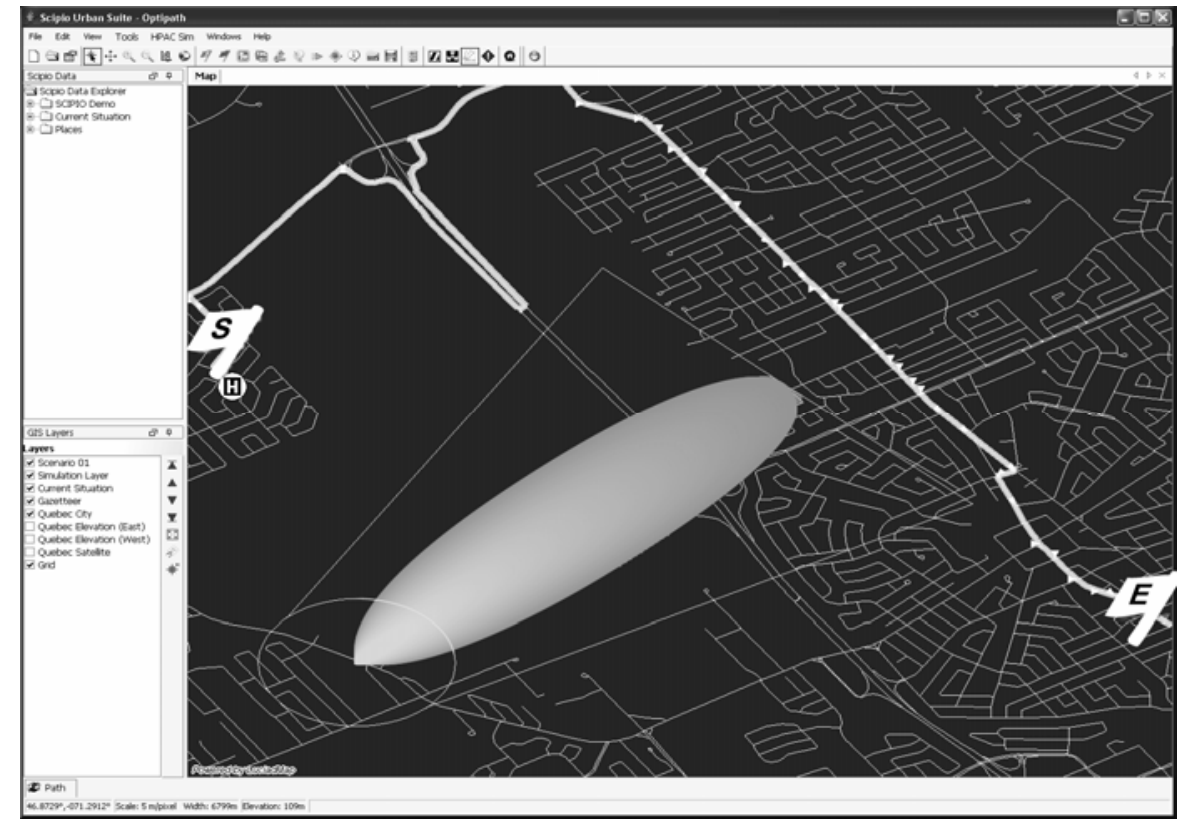

Figure 6 Path used when predicted threat situation becomes current - Adaptive behavior

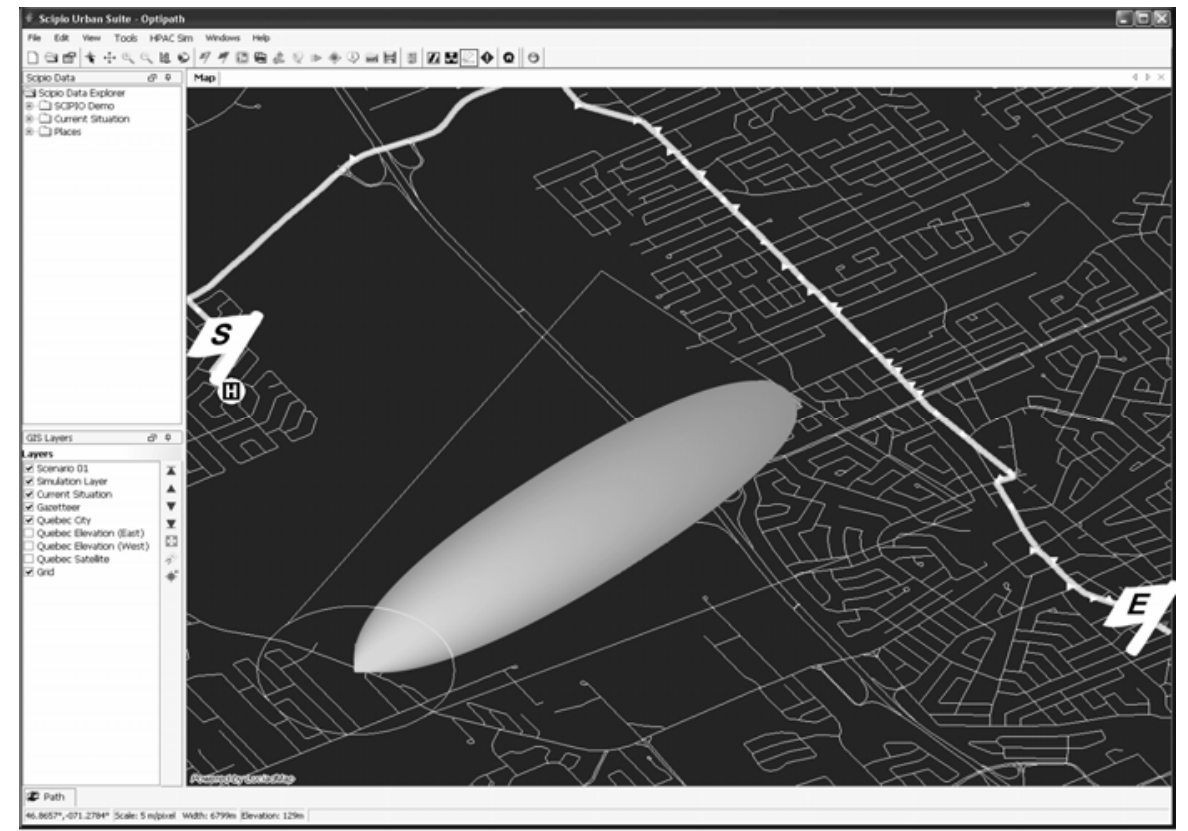

Figure 7 Path used when predicted threat situation becomes current Anticipative behavior

In Figure 6, the vehicle is in adaptive mode and travels along its original planned path (Figure 4). However, at the time of the predicted dispersion of the 
toxic cloud, the predictive information is still active in the database which means that it has been confirmed or that no other information contradicting it has been received. Thus it is displayed by the system and the vehicle must then assume that this information is now factual. The system reacts accordingly by readjusting the path for the vehicle with adaptive behavior in order to turn back before entering the cloud threat area by automatically identifying the next intersection or turning point (in this case a U-turn authorization point included in the map shape file) and computing again the path to the destination starting from this point. On the other hand, if the vehicle was operating in anticipative mode it doesn't need to readjust its path since the system had already taken into account the future threat from the beginning as shown in Figure 7.

It should be noted that if at any time, new information about a threat is entered in the system or threat information currently active in the system is modified or removed, the threat agents will automatically check if path updates are required. The difference lies in the nature of the information; if the new threat information is predictive then only paths in anticipative mode will be examined. Another important point to raise is that though it is not shown in the simple example above, predicted information usually comes as a set of discretized estimates ahead in time. In the case where the time interval between estimates is not close enough the system will perform interpolation in order to refresh the current situation at a reasonable rate or at specific time values. What this means is that if we have a few predictions about toxic cloud dispersion which are separated by large intervals in time it could be the case that a vehicle operating in adaptive mode will be caught right in the middle of the cloud, without having been redirected by the system when one of the predictions becomes factual. To avoid this, the system looks at the dispersion predictions and finds a rate at which the predictions must be interpolated (or queried for interpolation) according to the vehicle estimated travel speed and rate of dispersion. Accordingly, the system can deduce factual information from predicted one and use it in a timely manner to react as soon as the threat possibly intersects the path.

Table 3 lists the main system parameters and estimated results for the three situations in this scenario. Note that even if the quickest path is requested, the emergency behavior is not very urgent.

Table 3 First scenario main parameters and estimated results

\begin{tabular}{|c|c|c|c|}
\hline $\begin{array}{l}\text { Parameters and } \\
\text { results }\end{array}$ & $\begin{array}{l}\text { No threat info - } \\
\text { Adaptive \& } \\
\text { Anticipative }\end{array}$ & $\begin{array}{c}\text { Threat info - } \\
\text { Adaptive }\end{array}$ & $\begin{array}{l}\text { Threat info - } \\
\text { Anticipative }\end{array}$ \\
\hline Distance (km) & 1,72 & 2,29 & $\overline{1,82}$ \\
\hline Path optimization & Quickest & Quickest & Quickest \\
\hline Time (min, sec) & 1'08', & 2' 21 '” & 1'58', \\
\hline Threat tolerance & Safest & Safest & Safest \\
\hline $\begin{array}{l}\text { Threat } \\
\text { responsiveness }\end{array}$ & N.A. & Adaptive & Anticipative \\
\hline $\begin{array}{l}\text { Emergency } \\
\text { behavior }\end{array}$ & Normal & Normal & Normal \\
\hline
\end{tabular}


Comparing the three situations it is clear that the adaptive response is likely to be more expensive in terms of distance and time, depending on how quick the threat is recognized. On the other hand, the anticipative response is affected by the threat model behavior estimation. If the threat information is not available then the path computed is the quickest but it will likely lead the vehicle into the toxic cloud.

7.2 Scenario 2 - Evacuating victims from an incident location with alternative destinations

In this scenario, a number of injured people must be evacuated following an incident and in the presence of a nearby threat. After the victims have been examined by means of telemedicine and the preliminary injuries evaluation carried out (e.g., on-site resuscitation and stabilization required before transport, treatment without active resuscitation, or does not need treatment before transport, Cabrera et al. 2001), an on-site mobile telemedicine unit must evacuate the victims taking the threat evolution into account.

The map used for this scenario shows a different city than in the previous scenario. Two alternatives are illustrated in Figure 8: an evacuation path to a hospital but passing near the threat, or an evacuation path to a relief center in the opposite direction. These alternatives are specified in the scenario by selecting two end points (E1 and $E 2$ respectively) instead of one ${ }^{11}$.

\footnotetext{
${ }^{11}$ In our examples we used gazetteer locations for the cities that are added as a layer in the GIS and can be selected by a simple click when entering start and end points. Such locations are commonly available as text or shape files and when they are not, the system allows the user to enter coordinate points associated to hospitals, police stations, chemical plants etc. from any knowledgeable source by simply clicking on the map on the required points.
} 


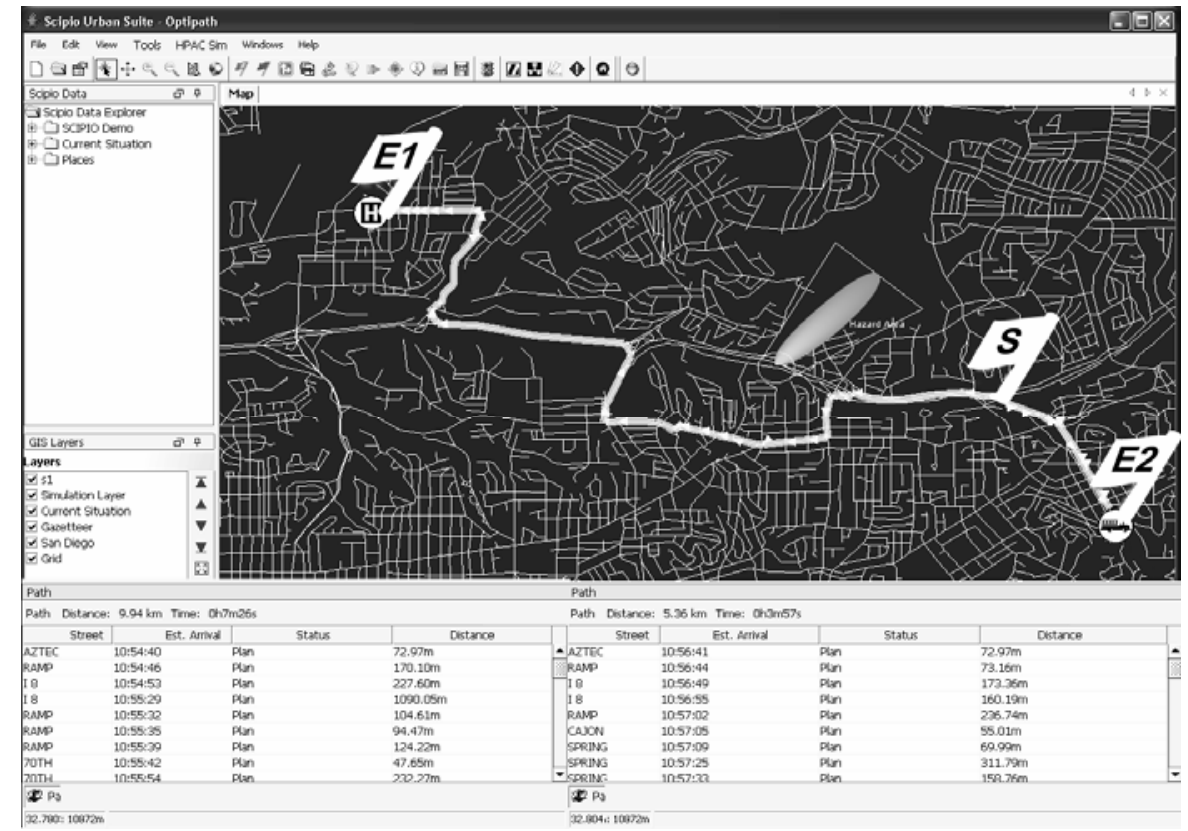

Figure 8 An emergency vehicle is being suggested two paths: one to a hospital (left side, E1), and the second to a closer relief station (E2)

During evacuation, the mobile telemedicine unit will not go back forcedly to the hospital it came from, needing to solve the optimal navigation problem again. The user will view and evaluate then the possible solutions, in terms of coherent destination for the victim, distance traveled and required time. Table 4 lists the main system parameters and estimated results for the two situations in this scenario.

Table 4 Second scenario main parameters and estimated results

\begin{tabular}{lcc}
\hline \multicolumn{1}{c}{$\begin{array}{c}\text { Parameters and } \\
\text { results }\end{array}$} & $\begin{array}{c}\text { To Hospital } \\
\text { E1 }\end{array}$ & $\begin{array}{c}\text { To Relief center } \\
\text { E2 }\end{array}$ \\
\hline Distance (km) & 9,94 & 5,36 \\
Path optimization & Quickest & Quickest \\
Time (min, sec) & 7' 26'’ & 3' 57'’ \\
Threat tolerance & Safest & Safest \\
Threat responsiveness & Anticipative & Anticipative \\
Emergency behavior & Very urgent & Very urgent \\
\hline
\end{tabular}

In this case, two optimal paths are proposed without recommending a particular one. Victims will be evacuated then, taking into account the preliminary injuries evaluation, the emergency response priorities, the resources management, and the availability of either equipped hospitals or advance primary care sites.

\subsection{Scenario 3 - Choosing the right telemedicine unit}

When several telemedicine units are available from different locations it is necessary to quickly decide which one should be sent to the location where 
victims need assistance. Figure 9 illustrates the system response, using a threat in another position, mobile units from three hospitals (S1,S3 and S4) and a relief station (S2) near the threat zone, besides all the scenario constraints.

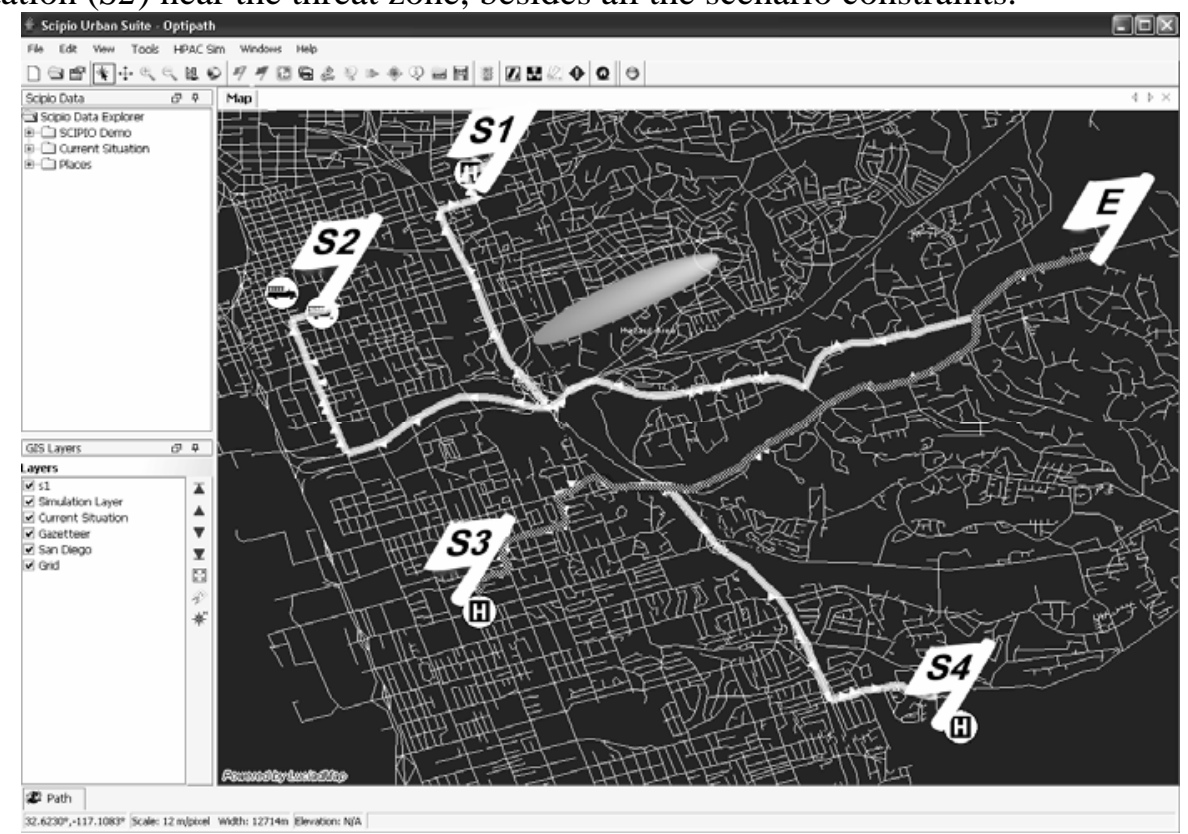

Figure 9 Suggestion of four paths according to starting points and rescue vehicle

Possibilities for the four mobile telemedicine units are evaluated and displayed. The best alternative is suggested. Table 5 lists the main system parameters and estimated results concerning the four situations of this scenario.

Table 5 Third scenario main parameters and estimated results

\begin{tabular}{|c|c|c|c|c|}
\hline $\begin{array}{l}\text { Parameters } \\
\text { and results }\end{array}$ & $\begin{array}{c}\text { From Hospital } \\
\text { S1 }\end{array}$ & $\begin{array}{c}\text { From Relief } \\
\text { center } S 2\end{array}$ & $\begin{array}{c}\text { From } \\
\text { Hospital } S 3\end{array}$ & $\begin{array}{c}\text { From Hospital } \\
\text { S4 }\end{array}$ \\
\hline Distance (km) & 5,16 & 5,31 & 4,61 & 5,58 \\
\hline $\begin{array}{l}\text { Path } \\
\text { optimization }\end{array}$ & ckest & & uickest & uickest \\
\hline $\begin{array}{l}\text { Time } \quad \text { (min, } \\
\text { sec) }\end{array}$ & 4' 59', & 4' 10', & 3' 32 '’ & $6^{\prime} 11^{\prime \prime}$ \\
\hline $\begin{array}{l}\text { Threat } \\
\text { tolerance }\end{array}$ & Safest & Safest & Safest & Safest \\
\hline $\begin{array}{l}\text { Threat } \\
\text { responsiveness }\end{array}$ & Anticipative & Anticipative & Anticipative & Anticipative \\
\hline $\begin{array}{l}\text { Emergency } \\
\text { behavior }\end{array}$ & Normal & Normal & Normal & Normal \\
\hline
\end{tabular}

The path starting from hospital at $S 3$ seems the best path because under the same conditions, it minimizes the distance and time traveled by the rescue unit. On the other hand, one could argue that the first portion of this path is closest to 
the threat than the first portion of the path starting from hospital at $S 4$ and it is thus more sensitive to the information accuracy on the threat location (reduced safety margin) on this path segment.

\subsection{Scenario 4 - Choosing the right hospital}

As in the second scenario, following the telemedicine examination and the preliminary injuries examination, the victims' evacuation path should be decided. At this point, the CCP is expected to have an estimated waiting time sent by the hospitals, besides information about specific equipment availability (simulated information in this example). The optimal evacuation path can be then calculated with these supplementary constraints. Figure 8 depicts the situation using two of the four hospital selection factors: existence of an emergency room and waiting time.

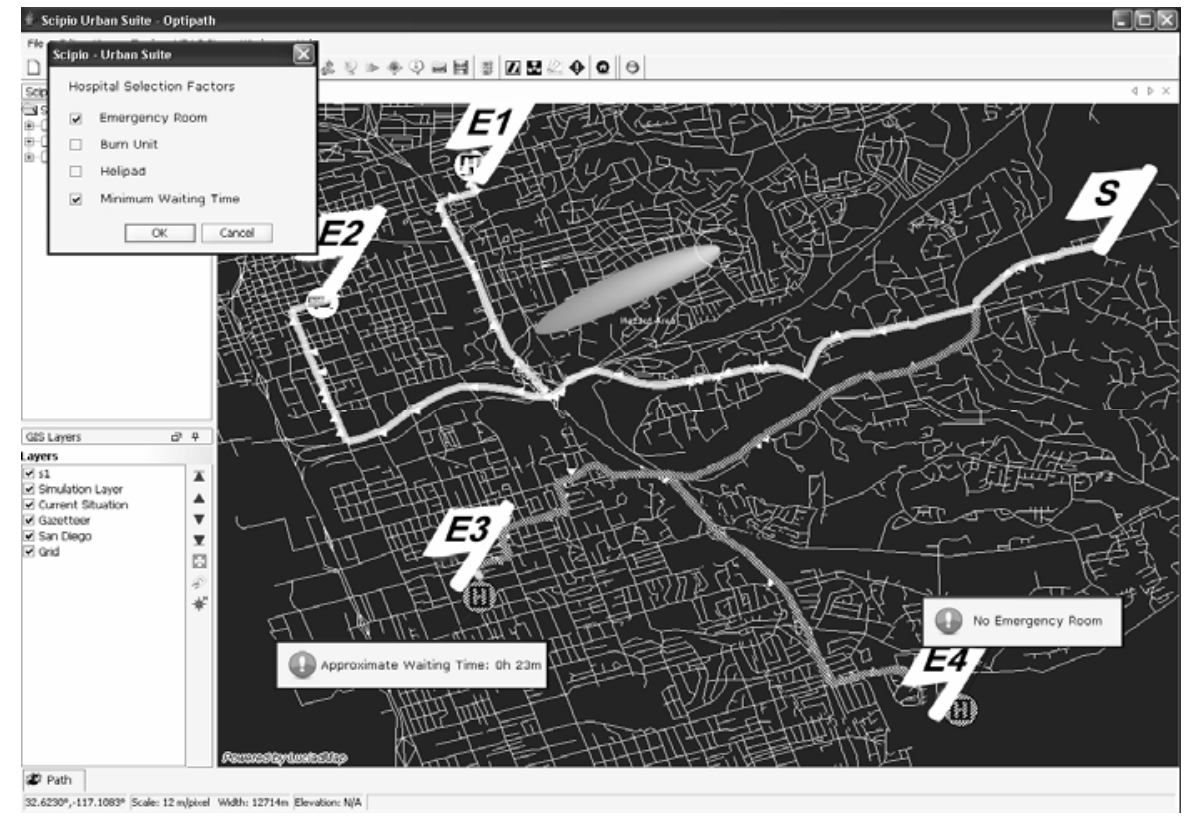

Figure 10 Suggestion of four paths according to available resources at destination

Four possibilities are evaluated and displayed. The best alternatives (first and second) are suggested. Table 6 lists the main system parameters and estimated results related to the four situations of this scenario. 
Table 6 Fourth scenario main parameters and estimated results

\begin{tabular}{|c|c|c|c|c|}
\hline $\begin{array}{c}\text { Parameters and } \\
\text { results }\end{array}$ & $\begin{array}{c}\text { To Hospital } \\
E 1\end{array}$ & $\begin{array}{l}\text { To Relief } \\
\text { center } E 2\end{array}$ & $\begin{array}{c}\text { To Hospital } \\
\text { E3 }\end{array}$ & $\begin{array}{c}\text { To Hospital } \\
E 4\end{array}$ \\
\hline Distance (km) & 5,16 & 5,31 & 4,61 & 5,58 \\
\hline $\begin{array}{l}\text { Path } \\
\text { optimization }\end{array}$ & Quickest & Quickest & Quickest & Quickest \\
\hline Time (min, sec) & 4' 59', & 4' 10', & 3'32'” & 6' 11'” \\
\hline $\begin{array}{l}\text { Threat } \\
\text { tolerance }\end{array}$ & Safest & Safest & Safest & Safest \\
\hline $\begin{array}{l}\text { Threat } \\
\text { responsiveness }\end{array}$ & Anticipative & Anticipative & Anticipative & Anticipative \\
\hline $\begin{array}{l}\text { Emergency } \\
\text { behavior }\end{array}$ & Normal & Normal & Normal & Normal \\
\hline Waiting time & $10^{\prime}$ & $15^{\prime}$ & $23{ }^{\prime}$ & $533^{\prime}$ \\
\hline $\begin{array}{l}\text { Emergency } \\
\text { room }\end{array}$ & yes & yes & yes & No \\
\hline
\end{tabular}

According to the applied selection criterion waiting times for hospital 3 and 4 are rather long, while emergency room in hospital 4 is not available. Two alternative optimal destinations, hospital at E1 and the relief center (E2) are proposed, matching the stated needs. Thereafter, it will be up to the CCP to decide how to proceed. Nevertheless, as emergency response progresses, hospital selection criteria become more difficult or impossible to satisfy. The system will list the different possible paths without selecting the optimal one, and indicating as in the example, the most penalizing constraints.

In all the scenarios, the path optimization criteria used was "Quickest" and threat tolerance was set to "Safest". These settings actually correspond to specific pre-defined weight combinations of the variables in the cost function. The system provides a standard interface with a list of such pre-defined labeled settings as well as an advanced cost function interface that allows the user to precisely select the variables of the cost function and their relative weight. In the context of the examples presented, "Quickest" path optimization and "Safest" threat tolerance criteria mean that the system will search for the fastest path that avoids all threats. Other threat tolerance settings may have produced a path that does enter an area under threat influence. The emergency behavior setting as "normal" indicates that though the path optimization criteria is "fastest", the vehicle is constrained to follow the speed limits (or less if traffic is heavy) on each road segment and cannot take one-way segments against incoming traffic. Setting the behavior to very urgent would have made the system look for a path considering that the vehicle may travel at a speed up to its MAS on each road segment and that it may use one-way segments.

Response time for all the path finding computations is quasi-immediate because the whole network graph representation is in memory, the number of threats and vehicles is limited and communications with external systems are restricted to a minimum (regular updates from the weather Web Service provider). Performance is mostly subjected to the processing of requests by 
external systems. This is why the algorithms implemented mostly rely on the system's memory and database instead of directly querying external sources of information. Updates in the database from external information are managed by the CIC component not the optimization one. Increasing the rate at which information is refreshed in the database would obviously add more processing efforts to recompute existing paths given an updated situation. Another situation that could affect performance is when we have a very large network that has been partitioned in smaller ones. The problem to be solved spans a number of smaller networks that often may require switching graphs in memory to complete the computations. These graphs must be read from the database which is a much slower operation than direct memory access.

\section{Discussion and conclusion}

The system presented in this paper is capable of managing a dynamic environment with multiple sources of information. More specifically, it can adjust in a timely manner the trajectory of a mobile telemedicine unit operating in an unstable environment that involves fixed and dynamic obstacles and threats. Threat management is supported by allowing input from different threat modeling and prediction systems in addition to basic capacities for user threat input information and processing. Impact of actual and predicted threats on the required vehicle operation are assessed by considering vehicle/crew capabilities with respect to optimization parameters specified in a configurable cost function. Illustrative examples with a toxic cloud were chosen because of their complexity compared to fixed obstacles such as road blocks or other potentially harmful agents (e.g., flood, hostile neighborhood). Trajectory corrections in response to fixed and mobile point threats are likely to be obtained using fewer parameters than mobile area threats. Predictive information can also be taken into account or ignored by the system by considering adaptive or anticipative response. Though the examples presented occur in an urban theater of operations, larger scale environments (regional, state) can also be considered if the underlying map data is available. In some cases of larger environments the map data may be so huge (i.e., several hundreds of thousand of edges) that the corresponding graph will be too large for computations in computer memory. Since the graph is persisted in the database it is always possible to perform the computations on a very large graph by querying the database. This solution has a major impact on the performance of the system compared to operations in memory. A better approach is map pre-processing to filter out road segments of less importance or to partition it into several smaller maps. The system's network topology analysis toolbox include functionalities to analyze and find such decomposition into smaller components according to different criteria (e.g., loosely connected clusters) and a tool (the GeoWorkshop) to filter, separate or merge such components into individual map files.

The system includes a complete GIS component with all related functionalities for managing and displaying information layers that includes all the usual functionalities of major GIS systems (line of sights, spatial analysis etc.). Other GIS or GIS-relatedsystems are (or have been) used for disaster management e.g., 
ESRI ArcGIS Spatial Analyst ${ }^{12}$, GeoConcept ${ }^{13}$, but they do not come as an integrated application with multi-source interoperability and optimization metacontrol. Our application is not intended as a substitute for a GIS but rather a threat management, optimization and decision-aid system that includes a GIS component. It supports connection protocols to different external information source providers for weather, traffic, geographic features, threat modelization and dispersion prediction data in addition to allowing the user to input its own data. Deployment configurations supported include large distributed network, fully operational standalone system hosted on portable computers and limited functionalities access on Web enabled devices.

The external sources of information incur inherent limitations on the system (availability of required information, communications availability, bandwidth and throughput, processing time, reliability of the external information provider system and validity/accuracy of the data provided). These limitations are somehow mitigated to a certain extent by allowing the system to operate in standalone configuration (at the CCP or in the mobile unit), supporting direct information input by the user (weather, traffic, threats, obstacles), incorporating previous knowledge in its database and performing processing on its own e.g., extracting geographic features, computing ATP-45 warning areas, running whatif scenarios.

Other current limitations include the fact that the provided paths are optimal only under a number of conditions which are seldom met in real world operations: perfect timing of the vehicle progress with respect to the plan, exactness of obstacles and threat information and predictions. In order to be satisfied, these conditions would require having constant access to perfectly accurate and timely information by constantly querying information providing sources and immediately obtaining results, both of which cannot be reasonably achieved. In real conditions, the defined optimal path would not be necessarily pursued by the telemedicine unit as expected, forcing to re-plan the path if time discrepancies between actual and planned times do not agree with the thresholds, adding to the issues concerning geographic-aware systems use for disaster decision support (Zerger and Smith 2003). For that reason, time discrepancies threshold values must be large enough to avoid constant path re-planning, yet small enough not to allow a path where actual times of passage will be too far from the optimal (unfeasible) path planned values. Following a planned optimal path with large discrepancy between planned and actual times of passage could lead directly to an area under threat that otherwise would have not been encountered. In the simulated scenarios, we have used several constant threshold values and observed, as expected, that as the number of dynamic events and rate of changes in the situation increase the smaller the thresholds must be set and, conversely, few dynamic events and a slower pace of changes allow for larger tolerance thresholds.

\footnotetext{
${ }^{12}$ http://www.esri.com/software/arcgis/extensions/spatialanalyst/index.html

${ }^{13}$ http://www.geoconcept.com/?74/-Rescue-Defence-
} 
Some of the limitations indicated are currently being addressed and the system is likely to show improvements on these aspects in future releases. For instance, we are working on better ways to process information on road intersections with control signs and modeling of vehicle turns at intersections. The path planning process should also allow vehicles to stop and wait for a moving threat to pass away (currently, this is partially addressed by permitting to include a number of optional way points along the path).

Several other enhancements are under development: providing paths for properly equipped personnel to rescue victims within threat areas e.g., firemen, mobile decontamination stations. In the current implementation, it is possible to select a destination lying within a hazard or contaminated area. However, the system needs information on the capabilities (protective equipment) of the vehicle and personnel with respect to the threat. In absence of such information, the threat tolerance level or cost function can be parameterized to account for a level of possible casualties among the crew but no provision is currently available to take into account the impact on the victims. We are also working on the management of simultaneous incident sites within the same scenario (at present, multiple incidents and the associated routing paths can be displayed simultaneously but only as part of different scenarios) and on the deployment of sensors at specific locations and within specific time windows to provide measurement of toxic material dispersion (needed by dispersion models to track and update predictions of toxic cloud dispersion). Other issues investigated include the possibility to adapt and make the system available on other mobile tele-security GPS/GIS devices such as Medical Intelligence Technologies Inc. Urgentys ${ }^{14}$ device and optimization of graph management in memory for large partitioned graphs.

Mobile telemedicine adds a displacement dimension to conventional telemedicine system setups, deployed at fixed places. The proposed navigation under threat validation demonstrates the system capability to forecast the required trajectory adjustments, decided as a function of the threat assessed impact, as well as the inferred threat tolerance and emergency level. The available data set is processed using a weighted attributed relational graph that assembles the scenario data, in order to optimize the trajectory according to the inputs. It applies dynamic knowledge management of a navigation rules database, to decide graph weights changes, and a cost function that evaluates information evolution through time, to reflect the new situation, processing a lot of information within a very short time.

\section{Acknowledgments}

This work was funded by Defence R\&D Canada grant no.1430JM12PM. The authors wish to thank Richard Grenier from Thales Canada for his support on this project.

\footnotetext{
${ }^{14}$ http://en.medicalintelligence.ca/MEDIA/Accueil/PDF/060108_EN_ProductTheColumb aBracelet_209.pdf
} 


\section{References}

Ahn, C.W., Ramakrishna, R.S., Choi, I.C., and Kang, C.G. (2002) 'Neural network based near-optimal routing algorithm', Proc. ICONIP02, pp. 1771-1776.

Benner, T., Schachinger, U., and Nerlich, M. (2004) 'Telemedicine in Trauma and Disasters - From War to Earthquake: Are we Ready', Studies in Health Technology and Informatics, vol. 104, pp. 106-115.

Botea, A., Müller, M., and Schaeffer, J. (2004) 'Near Optimal Hierarchical PathFinding’, Journal of Game Development, vol. 1, no. 1, pp. 7-28.

Bull, M. (2004) 'SAFE High Level Design', prepared for Defence Science and Technology Laboratory by RiskAware, Bristol.

Cabrera, M.F., Arredondo, M.T., Rodríguez, A., Quiroga, J. (2001) 'Mobile Technologies in the Management of Disasters: the Results of a Telemedicine Solution', Proc. American Medical Informatics Association Symposium, pp. 8689.

Chakroborty, P. and Wivedi, T. (2002) 'Optimal Route Network Design for Transit Systems Using Genetic Algorithms’, Engineering Optimization, vol. 34, no. 1, pp. 83-100.

Chan, T.C., Killeen, J., Griswold, W., and Lenert, L. (2004) 'Information Technology and Emergency Medical Care During Disasters', Academic Emergency Medicine, vol. 11, pp. 1229-1236.

Chu, Y. and Ganz, A. (2004) 'A Mobile Teletrauma System Using 3G Networks', IEEE Transactions on Information Technology in Biomedicine, vol. 8, no. 4, pp. 456-462.

Diez, H. (2002) 'SatCom Systems for Health and Medical Care', 34th COSPAR Scientific Assembly, p.IAA-1-4-02IAF.

DTRA (2005) 'The Hazard Prediction and Assessment Capability (HPAC) User's Guide Version 4.4', prepared for Defense Threat Reduction Agency by Science Applications International Corporation, San Diego.

Eppstein, D., Galil, Z., Italiano, G.F., and Nissenzweig, A. (1997) 'Sparsification - A Technique for Speeding Up Dynamic Graph Algorithms', Journal of the Association for Computing Machinery, vol. 44, no. 5, pp. 669-696.

Feliciani, F (2003) 'Medical Care from Space: Telemedicine', European Space Agency Bulletin, no. 114, pp. 54-59.

Garshnek, V. and Burkle, F.M. (1999) 'Applications of Telemedicine and Telecommunications to Disaster Medicine: Historical and Future Perspectives', Journal of the American Medical Informatics Association, vol. 6, pp. 26-37.

Herscovici, N., Christodoulou, C., Kyriacou, E., Pattichis, M.S., Pattichis, C.S., Panayides, A., and Pitsillides, A. (2007) 'm-Health e-Emergency Systems: Current Status and Future Directions', IEEE Antennas and Propagation Magazine, vol. 49, no. 1, pp. 216-231. 
Istepanian, R., Jovanov, E., and Zhang, Y.T. (2004) 'Guest Editorial. Introduction to the Special Section on M-Health: Beyond Seamless Mobility and Global Wireless Health-care Connectivity', IEEE Transactions on Information Technology in Biomedicine, vol. 8, no. 4, pp. 405-414.

Korkmaz, T., and Krunz, M. (2001) 'Multi-Constrained Optimal Path Selection', Proc. IEEE INFOCOM 2001, vol. 2, pp. 834-843.

Kyriacou, E., Pavlopoulos, S., Berler, A., Neophytou, M., Bourka, A., Georgoulas, A., Anagnostaki, A., Karayiannis, D., Schizas, C., Pattichis, C., Andreou, A., and Koutsouris, D. (2003) 'Multi-purpose HealthCare Telemedicine Systems with Mobile Communication Link Support', Biomedical Engineering OnLine, 2:7, 12 pp.

Mackenzie CF, Xiao Y, Lam D, Hu P, and Oglivie C. (2005) 'Telemedicine in Emergencies', in Human and Organizational Dynamics in e-Health, Eds. D.C. Bangert and R. Doktor, Radcliffe Publishing, Oxford, pp. 249-267.

NATO (1987) 'Reporting Nuclear Detonations, Biological and Chemical Attacks, and Predicting and Warning of Associated Hazards and Hazards Areas', NATO Allied Tactical Publication 45B.

NATO (2005) 'The Joint C3 Information Exchange Data Model (JC3IEDM Main)', NATO Multilateral Interoperability Program, edition. 3.00, DMGW.

Pattichis, C.S., Kyriacou, E., Voskarides, S., Pattichis, M.S., Istepanian, R., and Schizas, C.N. (2002) 'Wireless Telemedicine Systems: an Overview', IEEE Antenna's and Propagation Magazine, vol. 44, no. 2, pp. 143-153.

Rasid, M.F.A. and Woodward, B. (2005) 'Bluetooth Telemedicine Processor for Multichannel Biomedical Signal Transmission via Mobile Cellular Networks', IEEE Transactions on Information Technology in Biomedicine, vol. 9, no. 1, pp. 35-43.

Ramalingam, G., and Reps, T. (1996) 'An Incremental Algorithm for a Generalization of the Shortest-Path Problem', J. Algorithms, no. 21, pp. 267-305.

Stentz, A. (1994) 'Optimal and Efficient Path Planning for Partially-Known Environments', Proc. IEEE Int'l Conf. on Robotics and Automation, vol. 4, pp.3310-3317.

Tachakra, S., Wang, X.H., Istepahian, R., and Song, Y.H. (2003) 'Mobile eHealth: The Unwired Evolution of Telemedicine', Telemedicine Journal and eHealth, vol. 9, no. 3, pp. 247-257.

Takizawa, M., Sone, S., Hanamura, K., and Asakura, K. (2001) 'Telemedicine System Using Computed Tomography Van of High-Speed Telecommunication Vehicle', IEEE Transactions on Information Technology in Biomedicine, vol. 5, no. 1, pp. 2-9.

Teich, J.M., Wagner, M.M., Mackenzie, C.F., and Schafer, K.O. (2002) 'The Informatics Response in Disaster, Terrorism, and War', Journal of the American Medical Informatics Association, vol. 9. no. 2, pp. 97-104. 
Trovato, K.I., and Dorst, L. (2002) 'Differential A*', IEEE Transactions on Knowledge and Data Engineering, vol. 14, no. 6, pp. 1218-1229.

Widya, I., Vierhout, P., Jones, V.M., Bults, R., van Halteren, A., Peuscher, J., and Konstantas, D. (2006) 'Telematic Requirements for Emergency and Disasters Response Derived from Entreprise Models', in M-Health: Emerging Mobile Health Systems, Eds. R. Istepanian, C.S. Pattichis, and S. Laxminarayan, Springer, USA, pp. 531-547.

Xiao, Y., Gagliano, D., LaMonte, M., Hu, P., Gaasch, W., Gunawadane, R., and Mackenzie, C. (2000) 'Design and Evaluation of a Real-Time Mobile Telemedicine System for Ambulance Transport', Journal of High Speed Networks, vol. 9, pp. 47-56.

Zerger, A. and Smith, D.I. (2003) 'Impediments to Using GIS for Real-Time Disaster Decision Support', Computers, Environment and Urban Systems, vol. 27, pp. 123-141. 